\title{
Error Estimation and Assessment of an Approximation in a Wavelet Collocation Method
}

\author{
Marco Schuchmann, Michael Rasguljajew \\ University of Applied Science, Darmstadt, Germany \\ Email: michael_050485@yahoo.com
}

Received January 17, 2013; revised February 25, 2013; accepted March 23, 2013

Copyright (C) 2013 Marco Schuchmann, Michael Rasguljajew. This is an open access article distributed under the Creative Commons Attribution License, which permits unrestricted use, distribution, and reproduction in any medium, provided the original work is properly cited.

\begin{abstract}
This article describes how to assess an approximation in a wavelet collocation method which minimizes the sum of squares of residuals. In a research project several different types of differential equations were approximated with this method. A lot of parameters must be adjusted in the discussed method here. For example one parameter is the number of collocation points. In this article we show how we can detect whether this parameter is too small and how we can assess the error sum of squares of an approximation. In an example we see a correlation between the error sum of squares and a criterion to assess the approximation.
\end{abstract}

Keywords: ODE; Sinc Collocation; Shannon Wavelet; Wavelet Collocation; Error Estimation

\section{Introduction}

In the wavelet theory a scaling function $\phi$ is used, which has properties that are defined in the MSA (multi scale analysis). Through the MSA we know, we can construct an orthonormal basis of a closed subspace $V_{j}$, where $V_{j}$ belongs to a sequence of subspaces with the following property:

$$
\cdots \subset V_{-1} \subset V_{0} \subset V_{1} \subset \cdots \subset L^{2}(R),
$$

$\left\{\phi_{j, k}(t)\right\}_{k \in Z}$ is an orthonormal basis of $V_{j}$ with

$$
\phi_{j, k}(t)=2^{j / 2} \phi\left(2^{j} t-k\right) .
$$

We use the following approximation function:

$$
y_{j}(t):=\sum_{k=k_{\min }}^{k_{\max }} c_{k} \cdot \phi_{j, k}(t), \text { with } \phi \in C^{1}(R) .
$$

$k_{\min }$ and $k_{\max }$ depend on the approximation interval $\left[t_{0}, t_{\text {end }}\right]$.

Now we can approximate the solution of an initial value problem $y^{\prime}=f(y, t)$ and $y\left(t_{0}\right)=y_{0}$ by minimizing the following function ( \|\| is the Euklid norm)

$$
Q(c)=\sum_{i=1}^{m}\left\|y_{j}^{\prime}\left(t_{i}\right)-f\left(y_{j}\left(t_{i}\right), t_{i}\right)\right\|^{2}+\left\|y_{j}\left(t_{0}\right)-y_{0}\right\|^{2} .
$$

For $m=\left|k_{\max }-k_{\min }\right|$ we get an equivalent problem:

$$
\begin{gathered}
y_{j}^{\prime}\left(t_{i}\right)=f\left(y_{j}\left(t_{i}\right), t_{i}\right), \text { with } i=1,2, \cdots, m \text { and } \\
y_{j}\left(t_{0}\right)=y_{0} .
\end{gathered}
$$

The advantage of calculating $c$ by minimizing $Q$ is that we can choose more collocation points $t_{i}$ as shown in the following example. In that case we apply the least squares method to calculate $c$. Many simulations had shown that if $Q_{\min }$ was very small then the approximation $y_{j}$ would be good. An even better criterion for a good approximation $y_{j}$ is $Q_{a}$ (see (3)). Moreover, the equations have been ill-conditioned in several examples.

Analogously we could use boundary conditions instead of the initial conditions. This method can be even used analogously for PDEs, ODEs of higher order or DAEs, which have the form

$$
F\left(y^{\prime}, y, t\right)=0 .
$$

If $y^{\prime}=f(y, t)$ is an ODE system, then we use the approximation function:

$y_{j}(t)$

$=\left(\sum_{k=k_{\min }}^{k_{\max }} c_{k, 1} \phi_{j, k}(t), \sum_{k=k_{\min }}^{k_{\max }} c_{k, 2} \phi_{j, k}(t), \cdots, \sum_{k=k_{\min }}^{k_{\max }} c_{k, n_{f}} \phi_{j, k}(t)\right)^{\mathrm{T}}$

For the $i$-th component of the solution $y$, we use the notation $y_{j}$ as usual. We use for the $i$-th component of $y_{j}$ the notation $y_{j}^{(i)}$, in order not to lead to a confusion 
with the approximation $y_{j}$ out of $V_{j}$, so it will be always distinguished whether the approximation $y_{j}$ or the $i$-th component of $y$ is used.

We use the collocation points $t_{i}$, with $t_{i}=t_{0}+i \cdot h$ and

$$
h=\frac{t_{\text {end }}-t_{0}}{m}\left(m \geq\left|k_{\max }-k_{\min }\right|\right) .
$$

Simulations have shown that even with $m<\left|k_{\max }-k_{\min }\right|$ we get good approximations.

For the assessment of the approximation we use the value $Q_{a}$, with

$$
Q_{a}=\sum_{i=1}^{m_{a}}\left\|y_{j}^{\prime}\left(\tau_{i}\right)-f\left(y_{j}\left(\tau_{i}\right), \tau_{i}\right)\right\|^{2}+\left\|y_{j}\left(t_{0}\right)-y_{0}\right\|^{2},
$$

$\tau_{i}=t_{0}+i \cdot h / a, m_{a}=a \cdot m$ and $a>1$ is an integer. For big $a$ we should weight $Q_{a}$ with $1 / a$.

\section{Remarks 1:}

1) We get

$$
Q_{\min }^{\left(k_{\max }^{(1)}\right)} \leq Q_{\min }^{\left(k_{\max }^{(2)}\right)}
$$

for $k_{\max }^{(1)}>k_{\max }^{(2)}$, because of:

$$
Q^{\left(k_{\max }^{(1)}\right)}\left(c_{k_{\min }}, \cdots, c_{k_{\max }^{(1)}}, 0, \cdots, 0\right)=Q^{\left(k_{\max }^{(2)}\right)}\left(c_{k_{\min }}, \cdots, c_{k_{\max }^{(1)}}\right)
$$

Analogously for smaller $k_{\min }$.

2) The sums in (1) and (3) could start with $i=0$, too.

3) $\left\|y_{j}\left(t_{0}\right)-y_{0}\right\|^{2}$ in (1) could also be used as a constraint if the initial value should be fulfilled. But in all good approximations, $\left\|y_{j}\left(t_{0}\right)-y_{0}\right\|^{2}$ was very small.

In the examples we use the Shannon wavelet. Although it has no compact support and no high order, in many examples and simulations we got a much better approximation than using other wavelets (f.e. Daubechies wavelets of order 5 to 8 ), even with a small $n$. The Meyer wavelet yields good results, too.

We even get a good extrapolation outside the interval $\left[t_{0}, t_{\text {end }}\right]$.

\section{Example 1:}

1) We use the following ODE

$$
y^{\prime}=-t \cdot y, y(0)=1 \text {. }
$$

The exact solution is $y(t)=\mathrm{e}^{-1 / 2 t^{2}}$.

We approximated the solution on the interval $[0,1]$ and chose $k_{\max }=-k_{\min }$, like in all examples.

With $Q_{a}$ we could see in all our simulations, if the approximation was good. We got a linear relationship between $\ln \left(Q_{2}\right)$ and $\ln ($ sse $)$. In Figure 1 we see the graph of a linear regression (with an $R$ squared of 0.991196) of $\ln (s s e)$ against $\ln \left(Q_{2}\right)$ with the points $\left(\ln \left(Q_{2}\right), \ln (\right.$ sse $\left.)\right)$, which have been calculated with different $j(j=0,1,2), k_{\max }=-k_{\min }\left(k_{\max }=15,20,25\right)$ and $m\left(m=k_{\max }, 2 k_{\max }, 3 k_{\max }\right)$ with the ODE and $I$ of the example 1.

sse is the mean squared error

$$
\text { sse }=\sum_{i=0}^{100}\left(y\left(\tilde{t}_{i}\right)-y_{j}\left(\tilde{t}_{i}\right)\right)^{2} \text { with } \tilde{t}_{i}=i / 100 .
$$

Now we see a regression table (Table 1) of $\ln ($ sse) on $\ln \left(Q_{2}\right)$, which shows a linear dependency in our example and the graph of the linear regression function.

Here is a graph of the regression function and the graphs of the functions $y_{i}$ and $y_{j}-y$ for $j=0, k_{\max }=15$ and $m=30$ on the approximation interval $[0,1]$ (see Figures 2 and 3) and on the interval $[-1,2]$ (see Figures 4 and 5). In Figures 4 and 5 we see that we get even a good extrapolation.

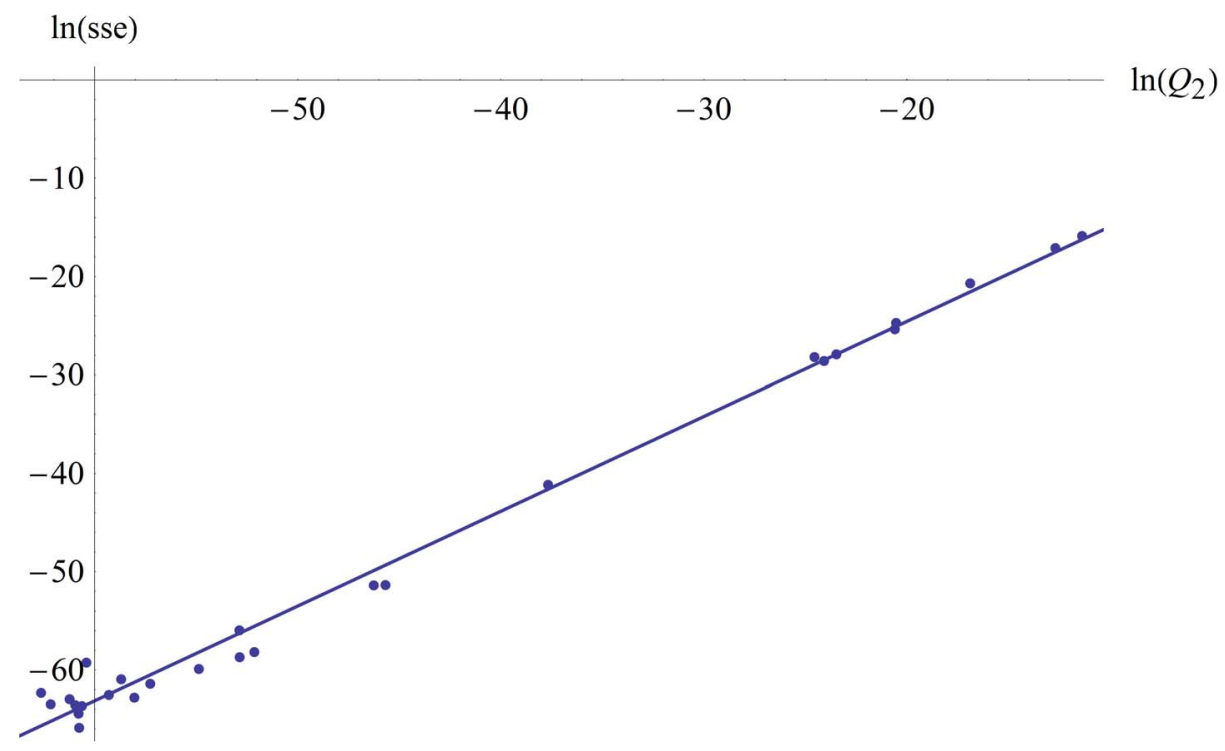

Figure 1. Linear regression plot of $\ln ($ sse $)$ against $\ln \left(Q_{2}\right)$. 


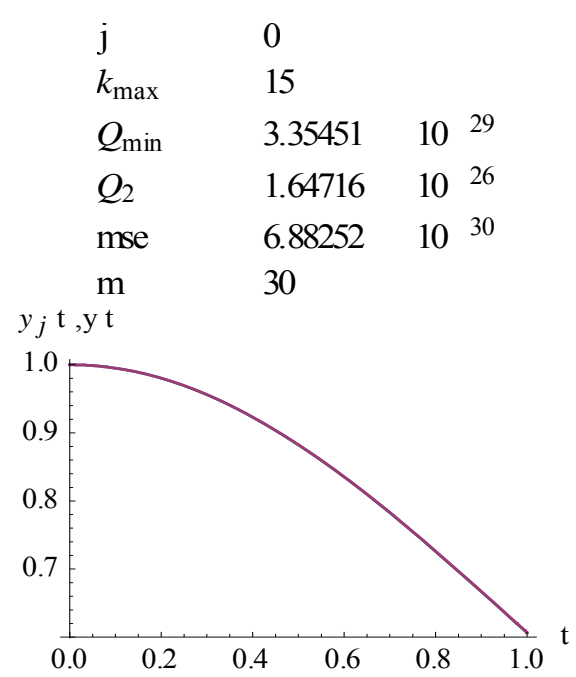

Figure 2. Graph of $y_{0}, k_{\max }=15, m=30$.

\begin{tabular}{|c|c|c|}
\hline $\mathrm{j}$ & 0 & \\
\hline$k_{\max }$ & 15 & \\
\hline$Q_{\min }$ & 3.35451 & $10^{29}$ \\
\hline$Q_{2}$ & 1.64716 & $10^{26}$ \\
\hline mse & 6.88252 & $10^{30}$ \\
\hline $\mathrm{m}$ & 30 & \\
\hline
\end{tabular}

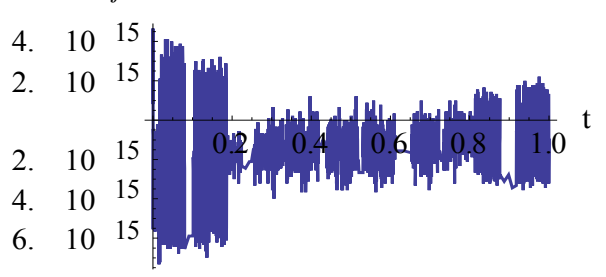

Figure 3. Graph of $y_{0}-y, k_{\max }=15, m=30$.

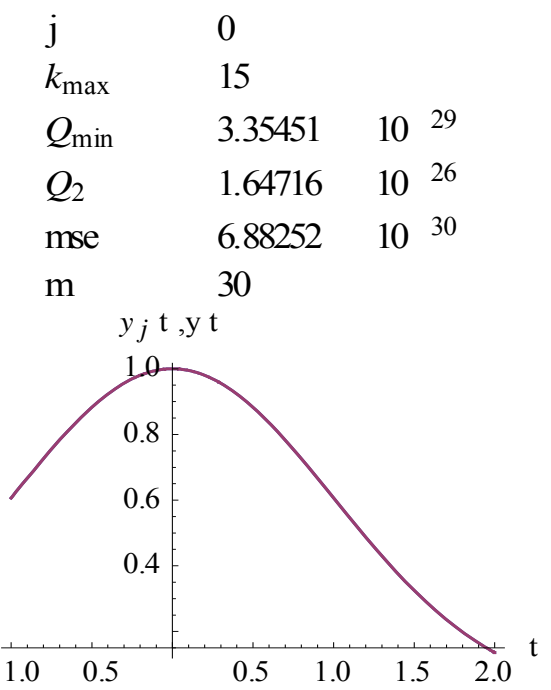

Figure 4. Graph of $y_{0}, k_{\max }=15, m=30$.

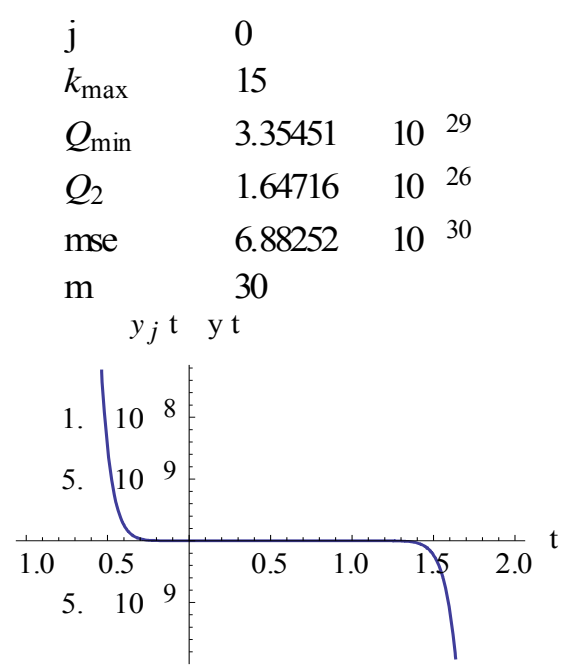

Figure 5. Graph of $y_{0}-y, k_{\max }=15, m=30$.

Table 1. Linear regression table of $\ln ($ sse $)$ on $\ln \left(Q_{2}\right)$.

\begin{tabular}{ccccc}
\hline & Estimate & SE & T Stat & P Value \\
\hline Intercept & -5.34843 & 0.882147 & -6.06297 & $2.46251 \times 10^{-6}$ \\
Slope & 0.962059 & 0.0181342 & 53.0523 & $3.2288 \times 10^{-27}$ \\
\hline
\end{tabular}

\section{Error Estimation and Assessment of the Approximation}

In the example we used the Shannon wavelet. For this wavelet we have additional information about the error in the Fourier space from the Shannon theorem. For a good approximation with a small $j$ the behavior of $Y(\omega)$ with growing $|\omega|$ is important, because (if $y_{i}$ is an orthogonal projection from $y$ on $V_{j}$ and $k_{\max }=-k_{\min }=\infty$ )

$$
\begin{aligned}
& y(t)-y_{j}(t) \\
& =\frac{1}{\sqrt{2 \pi}} \int_{-\infty}^{\infty} Y(\omega) \mathrm{e}^{\mathrm{i} \omega t} \mathrm{~d} \omega-\frac{1}{\sqrt{2 \pi}} \int_{-2^{j} \pi}^{2^{j} \pi} Y(\omega) \mathrm{e}^{\mathrm{i} \omega t} \mathrm{~d} \omega \\
& =\frac{1}{\sqrt{2 \pi}} \int_{-\infty}^{-2^{j} \pi} Y(\omega) \mathrm{e}^{\mathrm{i} \omega t} \mathrm{~d} \omega+\frac{1}{\sqrt{2 \pi}} \int_{2^{j} \pi}^{\infty} Y(\omega) \mathrm{e}^{\mathrm{i} \omega t} \mathrm{~d} \omega .
\end{aligned}
$$

With the Parseval theorem we get

$$
\left\|y-y_{j}\right\|_{L^{2}}=\sqrt{\int_{-\infty}^{-2^{j} \pi}|Y(\omega)|^{2} \mathrm{~d} \omega+\int_{2^{j} \pi}^{\infty}|Y(\omega)|^{2} \mathrm{~d} \omega},
$$

so

$$
\left\|d_{j}\right\|_{L^{2}}=\sqrt{\int_{-2^{j+1} \pi}^{-2^{j} \pi}|Y(\omega)|^{2} \mathrm{~d} \omega+\int_{2^{j} \pi}^{2^{j+1} \pi}|Y(\omega)|^{2} \mathrm{~d} \omega}
$$

With the Riemann-Lebesgue theorem we get:

$$
\lim _{j \rightarrow \infty}\left\|d_{j}\right\|_{L^{2}}=0
$$


For the approximation error the decay behaviour of the detail coefficients $d_{k}^{j}=\left\langle y, \psi_{j, k}\right\rangle$ is important:

$$
\begin{aligned}
\left\|y-y_{j}\right\|_{L^{2}} & =\left\|\sum_{s=j}^{\infty} d_{s}\right\|_{L^{2}}=\left\|\sum_{s=j}^{\infty} \sum_{k=-\infty}^{\infty} d_{k}^{s} \psi_{s, k}\right\|_{L^{2}} \\
& =\sqrt{\sum_{s=j}^{\infty} \sum_{k=-\infty}^{\infty}\left|d_{k}^{s}\right|^{2}}
\end{aligned}
$$

On the other side: we have got in many simulations with the Shannon wavelet better approximations (with the described collocation method) than with higher order wavelets.

\section{Remarks 2:}

1) For a theoretical multi resolution analysis we could consider $1_{I} \cdot y$ instead of $y$, because when $y$ is in $L^{2}(I)$ then $1_{I} \cdot y$ is in $L^{2}(R)$, if we need an approximation on $I$. Here $1_{I}$ is the indicator function of the interval $I$.

2) For interpolating wavelets there are a number of publications with error estimates and also for the approximation of the solutions of initial value problems and boundary value problems (for ordinary and partial differential equations) see $[1,2]$, as well as to the sinc collocation method (see [3-5]) with special collocation points ("sinc grid points", see [5]).

\section{Theorem 1 (for the decay behaviour):}

The wavelet $\psi$ has the order $p, y \in L^{2}(R)$, $y \in C^{r}(R)$ with $r<p$ and $y^{(r)}$ is Lipschitz continuous. Then exists a $c>0$ independent from $b$ with

$$
\left|W_{y}(a, b)\right| \leq c|a|^{r+3 / 2} .
$$

$W_{y}$ is the wavelet transform of $y$ with

$$
W_{y}(a, b)=|a|^{-1 / 2} \cdot \int_{-\infty}^{\infty} y(t) \cdot \overline{\psi\left(\frac{t-b}{a}\right)} \mathrm{d} t .
$$

A proof is in [6]. So we get for the detail coefficients an appraisal because

$$
\begin{aligned}
d_{k}^{j} & =\left\langle y, \psi_{j, k}\right\rangle=\int_{I R} y(t) \overline{2^{j / 2} \psi\left(2^{j} t-k\right)} \mathrm{d} t \\
& =W_{y}\left(2^{-j}, k 2^{-j}\right)
\end{aligned}
$$

and so

$$
\left|d_{k}^{j}\right|=\left|W_{y}\left(2^{-j}, k 2^{-j}\right)\right| \leq c \cdot 2^{-j \cdot(r+3 / 2)} .
$$

Now we saw that the decay of the detail coefficients depends on the order of a wavelet.

From the Gilbert-Strang Theory (see [7]) we know additionally an upper bound of the approximation error in dependency of the order $p$ : if the wavelet is of order $p$ then the approximation error has the order $O\left(2^{-j \cdot p}\right)$ if $\left\|y^{(p)}\right\|_{L^{2}}<\infty$ and (if $y_{j}$ is an orthogonal projection from $L^{2}$ on $V_{j}$ and $k_{\max }=-k_{\min }=\infty$ )

$$
\left\|y-y_{j}\right\|_{L^{2}} \leq C_{\phi} \cdot 2^{-j p} \cdot\left\|y^{(p)}\right\|_{L^{2}} .
$$

If a wavelet is of order $p$ the scaling function $\phi$ even has an interpolation property, because then we can construct the functions $t^{r}$ with $r=0,1, \cdots, p-1$ over a linear combination of $\phi(t-k)$ (see [7]). That's also a property of the so called interpolating wavelets. For interpolating wavelets we find error estimations in [8] and [9].

\section{Remarks 3:}

1) Error estimations for the sinc collocation with a transformation can be found in [4] and [5].

2) Although the approximation error is depended on the order of a wavelet in many simulations the Shannon wavelet led to much better approximations than Daubechies wavelets of higher order, if the approximation function $y_{j}$ was calculated by minimizing the sum of squares of residuals $Q$. Even when comparing the extrapolations the Shannon wavelet was significantly better.

The reason is, that we do not calculate an orthogonal projection on $V_{j}$ like in the appraisal above and the function $y$ is in general case not quadratic integrabel on $R$ (we consider only a compact interval $I$ ).

The following appraisal takes account of the fact that we calculate the approximation function by the minimization of $Q$. We first need a theorem, which follows from the Gronwall-Lemma.

\section{Theorem 2:}

Assumptions: we have a initial value problem $y^{\prime}=f(y, t)$ with $y\left(t_{0}\right)=y_{0}$ and

$$
\begin{aligned}
& \left\|y_{j}\left(t_{0}\right)-y\left(t_{0}\right)\right\| \leq \delta, \\
& \left\|y_{j}^{\prime}(t)-f\left(y_{j}(t), t\right)\right\| \leq M
\end{aligned}
$$

and

$$
\begin{gathered}
\left\|f(y(t), t)-f\left(y_{j}(t), t\right)\right\| \leq L \cdot\left\|y(t)-y_{j}(t)\right\| \\
\text { with } L>0 .
\end{gathered}
$$

Then we get for $t \geq t_{0}$ :

$$
\left\|y(t)-y_{j}(t)\right\| \leq \delta \cdot \mathrm{e}^{L\left(t-t_{0}\right)}+M / L \cdot\left(\mathrm{e}^{L\left(t-t_{0}\right)}-1\right)
$$

For a proof see [10].

\section{Theorem 3:}

With the assumptions from Theorem 2 we get (if $\left.y_{j}\left(t_{0}\right)=y_{0}\right)$ :

$$
\text { sse }=\sum_{i=1}^{\tilde{m}}\left\|y_{j}\left(\tilde{t}_{i}\right)-y\left(\tilde{t}_{i}\right)\right\|^{2} \leq(M / L)^{2} \cdot \underbrace{\sum_{i=1}^{\tilde{m}}\left(\mathrm{e}^{L\left(\tilde{t}_{i}-t_{0}\right)}-1\right)^{2}}_{=C_{L}}
$$

So we get the follow inequality for $\ln (s s e)$, which is used in the example 2 : 


$$
\ln (\text { sse }) \leq \ln \left(M^{2}\right)-\ln \left(L^{2}\right)+\ln \left(C_{L}\right)
$$

$\ln \left(M^{2}\right)$ depends on $y_{j}$ and $\ln \left(C_{L}\right)-\ln \left(L^{2}\right)$ only on the initial value problem and the collocation points. We write $\ln \left(M^{2}\right)$ instead of $2 \ln (M)$ because in example 2 we set $\ln \left(M^{2}\right)$ on the $x$-axes so we have a comparison with example 1 where we set $\ln \left(Q_{2}\right)$ on the $x$-axes.

\section{Remark 4:}

We get with $y_{j}\left(t_{0}\right)=y_{0}$

$$
Q_{\min }=\sum_{i=1}^{m}\left\|y_{j}^{\prime}\left(t_{i}\right)-f\left(y_{j}\left(t_{i}\right), t_{i}\right)\right\|^{2} \leq m \cdot M^{2} .
$$

If additionally $\left\|y_{j}^{\prime}\left(t_{i}\right)-f\left(y_{j}\left(t_{i}\right), t_{i}\right)\right\|=M$ for one (or more) $i \in\{1,2, \cdots, m\}$ we get:

$$
M \leq \sqrt{Q_{\min }} \leq \sqrt{m} \cdot M
$$

This is analogously right for $Q_{a}$ instead if $Q_{\min }$ with

$$
Q_{a}=\sum_{i=1}^{m_{a}}\left\|y_{j}^{\prime}\left(\tau_{i}\right)-f\left(y_{j}\left(\tau_{i}\right), \tau_{i}\right)\right\|^{2}
$$

and $\tau_{i}=t_{0}+i \cdot h / a, m_{a}=a \cdot m$ and an integer $a>1 . Q_{a}$ is an upper bound for $Q_{\min }: Q_{\min } \leq Q_{a}$. With $Q_{a}$ we could assess in all simulations the quality of an approximation and in linear regressions from $\ln ($ sse $)$ on $\ln \left(Q_{2}\right)$ we got in almost all simulations a $R^{2}$ ( $R$ squared) greater than 0.99 (see next example). Only if all approximations have been bad, then $R^{2}$ was less than 0.99 (but we still have a dependency). If $y_{j}$ is the exact solution, then $Q_{a}=0$. Because we get not only a approximation with points (we get a approximation function $y_{j}$ ) we must not calculate a second minimization for the calculation of $Q_{a}$

$\left\|y_{j}^{\prime}\left(t_{i}\right)-f\left(y_{j}\left(t_{i}\right), t_{i}\right)\right\|$ will be in general (for $i>0$ ) less than $M$, because we use the collocations points $t_{i}$ and so $\left\|y_{j}^{\prime}\left(t_{i}\right)-f\left(y_{j}\left(t_{i}\right), t_{i}\right)\right\|$ is very small at these points (see the next graphic). $Q_{\min }$ was in many good simulations less than $10^{-16}$.

In many simulation $\left\|y_{j}^{\prime}\left(t_{i}\right)-f\left(y_{j}\left(t_{i}\right), t_{i}\right)\right\|$ is relative big between to collocation points (or at the edge of $I$ if we start with $i=1$ in the sum (1)).

In Figure 6 we see the graph of

$$
d(t)=\left(y_{j}^{\prime}(t)-f\left(y_{j}(t), t\right)\right)^{2}
$$

in example 1 for $j=2, k_{\max }=10$ and $m=4$. Here a too small $m$ results in a very bad approximation.

We see that $Q_{\min }$ could be very small with a too small $m$, but $Q_{2}$ is very big here. In the graph we see that $d$ is very small at the collocation points $t_{i}=0.25 i$ but between them $d$ is very big. That's the reason because we could identify with $Q_{2}$ a worse approximation in any our simulations. On the other hand a big $Q_{\min }$ is an indicative of a too small $j$.

So we can approximate $M$ here with the maximum of $\left\|y_{j}^{\prime}\left(\tau_{i}\right)-f\left(y_{j}\left(\tau_{i}\right), \tau_{i}\right)\right\|$ at the points $\tau_{i}=t_{0}+i \cdot h / a$ with $a=2$ like we do it in the next example.

Now we want to apply the result from theorem 2. Furthermore we will see a correlation between an approximation of $\ln \left(M^{2}\right)$ and $\ln ($ sse $)$ in this example like we saw it before between $\ln \left(Q_{2}\right)$ and $\ln ($ sse $)$.

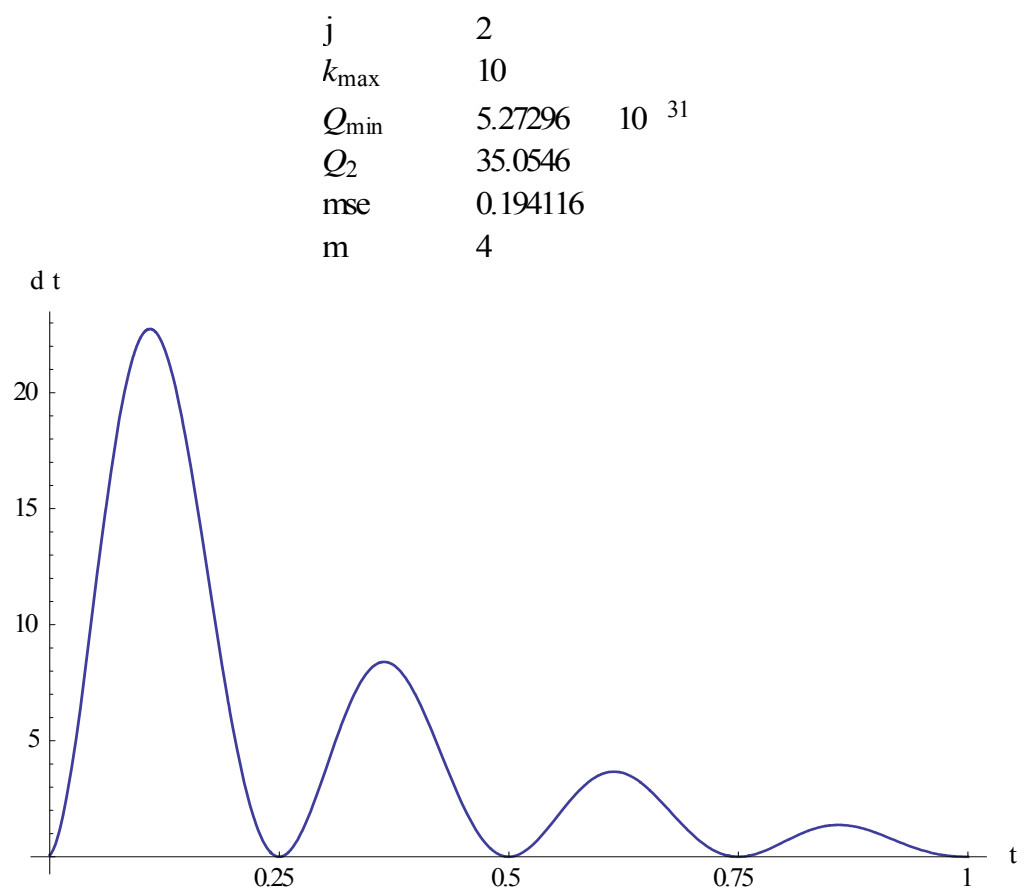

Figure 6. Graph of $d$. 


\section{Example 2:}

We use the initial value problem and the approximations with the different parameters $j, k_{\max }$ and $m$ of example 1. If $\delta>0$ than follows from theorem 2 (under the assumptions from this theorem):

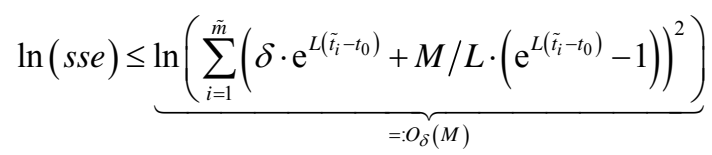

Here we get (see (6)):

$$
O_{0}(M)=\ln \left(M^{2}\right)-\ln \left(L^{2}\right)+\ln \left(C_{L}\right)
$$

We now apply a linear regression of $\ln ($ sse $)$ on $\ln \left(\hat{M}^{2}\right)$ with the approximation

$$
\hat{M}^{2}=\max \left\|y_{j}^{\prime}\left(\tau_{i}\right)-f\left(y_{j}\left(\tau_{i}\right), \tau_{i}\right)\right\|^{2},
$$

from $M^{2}$ with $\tau_{i}=t_{0}+i \cdot h / 2, i=0,1,2, \cdots, 200$ (the points from $Q_{2}$ beginning with $\left.i=0\right)$. sse and $O_{\delta}$ have been calculated with the points $\tilde{t}_{i}=i / 100$ (and the summation indices $i=0,1,2, \cdots, 100$ ).

Here is the regression table (Table 2) (with a $R$ squared of 0.986877).

In Figure 7 we see a graph from $O_{0}$ (in red), the graph of the regression function (in blue) and the regression points $\left(\ln \left(\hat{M}^{2}\right), \ln (s s e)\right)$. $\left\|y_{j}\left(t_{0}\right)-y\left(t_{0}\right)\right\|$ was not considered (this means we set $\delta=0$ ) because it was very small.

Here are the graphs of $O_{\delta}$ with $\delta=10^{-10}, \delta=10^{-15}$ and $\delta=0$ in Figure 8. In most simulations $\delta$ was less than $10^{-16}$.

Generally we can use

$$
\hat{M}_{a}^{2}=\max \left\|y_{j}^{\prime}\left(\tau_{i}\right)-f\left(y_{j}\left(\tau_{i}\right), \tau_{i}\right)\right\|^{2},
$$

$\tau_{i}=t_{0}+i \cdot h / a$ and $i=0,1, \cdots, a \cdot m$ (with an integer $a>1$ ) for an approximation of $M^{2}$. Here we know the following relation:

$$
\hat{M}_{a}^{2} \leq Q_{a}
$$

\section{Conclusions}

We defined a variable $Q_{a}$ with which you can evaluate an approximation. In many simulations and in the examples of this article we saw that we get good results with $a=2$. A linear relationship between $\ln (Q$,$) and$ $\ln ($ sse $)$ was shown in example 1 . It is also shown that the approximation can be used to extrapolate outside the approximation interval.

Using Theorem 2 we derive an estimate (see theorem 3). Then it is shown how to detect a too great step size using $Q_{2}$. In example 2 we show that the deduced estimate represents a straight line (in the coordinate svstem with $\ln \left(M^{2}\right)$ on the $x$-axes and $\ln ($ sse $)$ on the $y$ axes), which runs approximately parallel to the regression line (it is approximately parallel because the regression function is an estimation, theoretically it must be parallel because it cannot cross the upper bound line). In a research project we got analogous results in many

Table 2. Regression table of $\ln ($ sse $)$ on $\ln \left(\hat{M}^{2}\right)$.

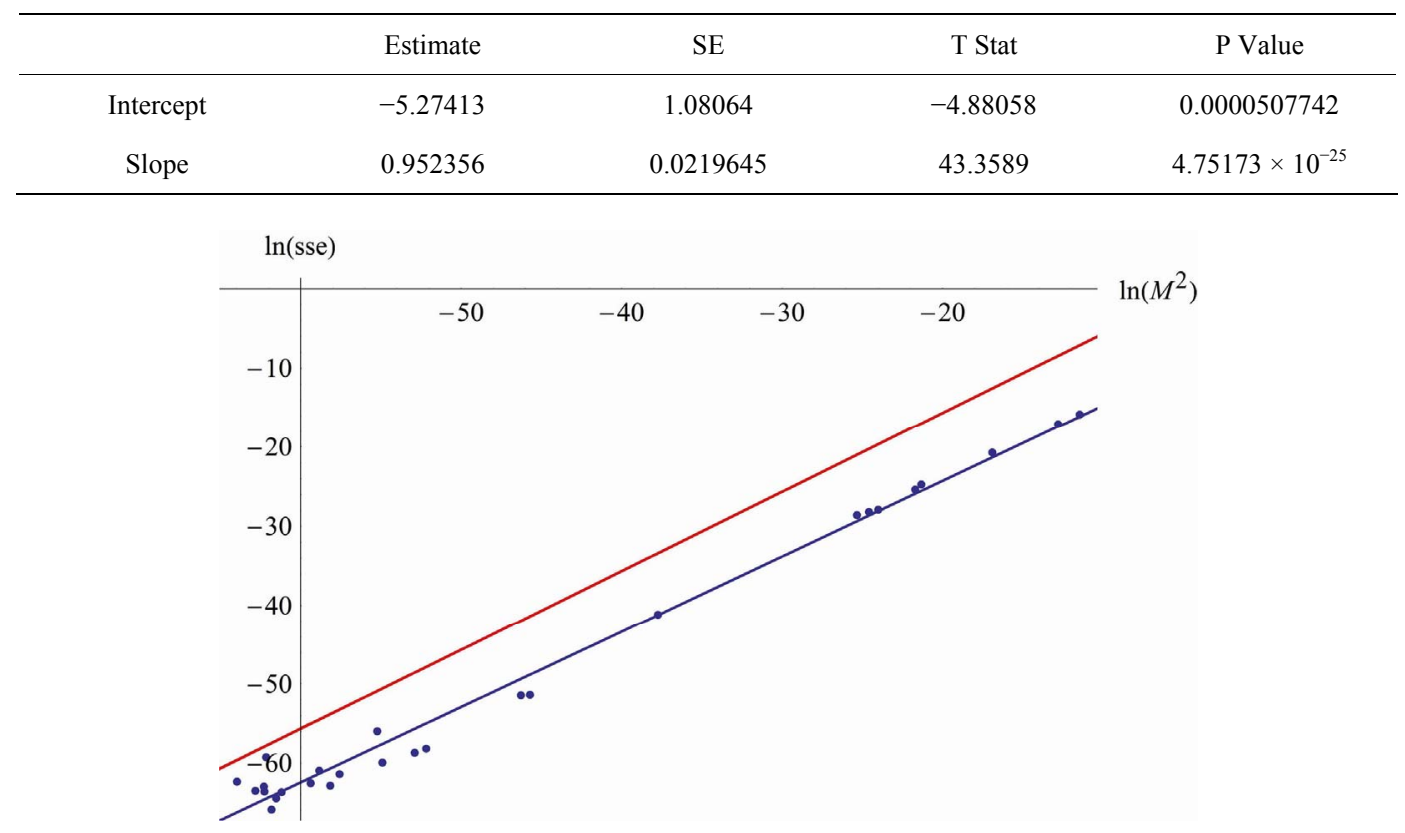

Figure 7. Linear regression plot of $\ln (s s e)$ against $\ln \left(\hat{M}^{2}\right)$. 


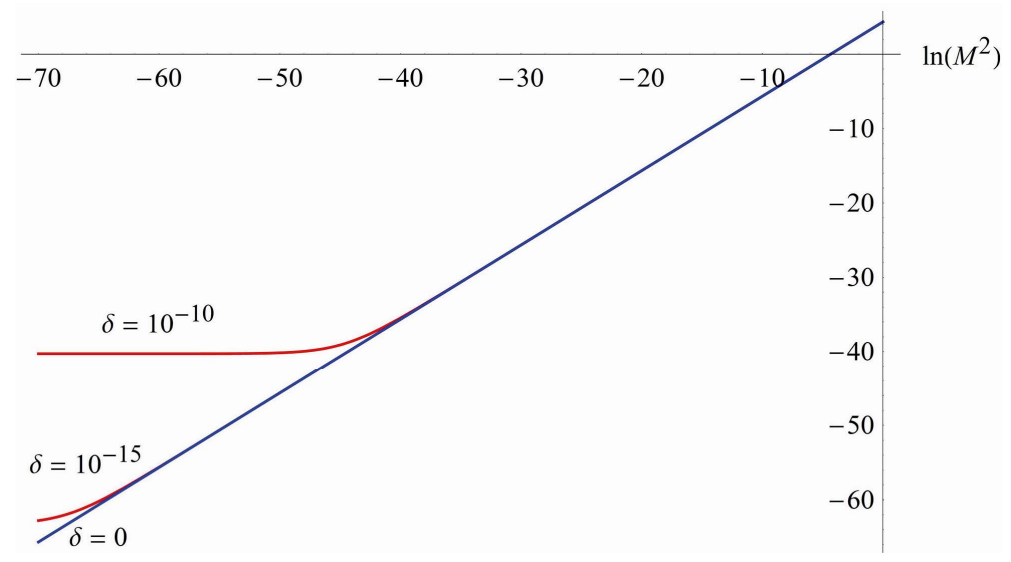

Figure 8. Graphs of $O_{\delta}$ with $\delta=10^{-10}, \delta=10^{-15}$ and $\delta=0$.

simulations. even with svstems and higher order odes.

It is shown that $M^{2}$ (the size of the estimate) can be approximated via $\hat{M}^{2}$, and this approximation has $Q_{2}$ as upper bound. The regression of the points

$\left(\ln \left(Q_{2}\right), \ln (\right.$ sse $\left.)\right)$ returns a slightly larger $R^{2}$ than the regression with the points $\left(\ln \left(\hat{M}^{2}\right), \ln (s s e)\right)$. As a consequence, $Q_{2}$ is well suited to assess, especially as you can estimate the approximation of $M^{2}$ with $Q_{2}$ and in $Q_{2}$ more information is included. Moreover we can compare $Q_{2}$ with $Q_{\min }$ to assess the approximation (see Figure 6).

\section{REFERENCES}

[1] O. V. Vasilyev and C. Bowman, "Second-Generation Wavelet Collocation Method for the Solution of Partial Differential Equations," Journal of Computational Physics, Vol. 165, No. 2, 2000, pp. 660-693. https://wiki.ucar.edu/download/attachments/41484400/va silyev1.pdf doi:10.1006/jcph.2000.6638

[2] S. Bertoluzza, "Adaptive Wavelet Collocation Method for the Solution of Burgers Equation," Transport Theory and Statistical Physics, Vol. 25, No. 3-5, 2006, pp. 339-352. doi:10.1080/00411459608220705

[3] T. S. Carlson, J. Dockery and J. Lund, "A Sinc-Collocation Method for Initial Value Problems," Mathematics of Computation, Vol. 66, No. 217, 1997, pp. 215-235.

\section{doi:10.1090/S0025-5718-97-00789-8}

[4] K. Abdella, "Numerical Solution of Two-Point Boundary Value Problems Using Sinc Interpolation," Proceedings of the American Conference on Applied Mathematics, Applied Mathematics in Electrical and Computer Engineering, 2012, pp. 157-162.

[5] A. Nurmuhammada, M. Muhammada, M. Moria and M. Sugiharab, "Double Exponential Transformation in the Sinc-Collocation Method for a Boundary Value Problem with Fourth-Order Ordinary Differential Equation," Journal of Computational and Applied Mathematics, Vol. 162, No. 2, 2005, pp. 32-50. doi:10.1016/j.cam.2004.09.061

[6] C. Blatter, "Wavelets-Eine Einführung," 2nd Edition, Vieweg, Wiesbaden, 2003.

[7] G. Strang, "Wavelets and Dilation Equations: A Brief Introduction," SIAM Review, Vol. 31, No. 4, 1989, pp. 614627. doi:10.1137/1031128

[8] Z. Shi, D. J. Kouri, G. W. Wie and D. K. Hoffman, "Generalized Symmetric Interpolating Wavelets," Computer Physics Communications, Vol. 119, No. 2-3, 1999, pp. 194-218. doi:10.1016/S0010-4655(99)00185-X

[9] D. L. Donoho, "Interpolating Wavelet Transforms," Technical Report 408, Department of Statistics, Stanford University, Stanford, 1992.

[10] E. Hairer and G. Wanner, "Solving Ordinary Differential Equations I: Nonstiff Problems," 2nd Edition, Springer, Berlin, 1993. 